\title{
Is there a path from cuprates towards room-temperature superconductivity?
}

\author{
I. Bozovic
}

Submitted to Quantum Studies: Mathematics and Foundations (2017)

August 2017

\author{
Condensed Matter Physics and Materials Science Department \\ Brookhaven National Laboratory
}

\author{
U.S. Department of Energy \\ USDOE Office of Science (SC), \\ Basic Energy Sciences (BES) (SC-22)
}

Notice: This manuscript has been authored by employees of Brookhaven Science Associates, LLC under Contract No. DE- SC0012704 with the U.S. Department of Energy. The publisher by accepting the manuscript for publication acknowledges that the United States Government retains a non-exclusive, paid-up, irrevocable, world-wide license to publish or reproduce the published form of this manuscript, or allow others to do so, for United States Government purposes. 


\section{DISCLAIMER}

This report was prepared as an account of work sponsored by an agency of the United States Government. Neither the United States Government nor any agency thereof, nor any of their employees, nor any of their contractors, subcontractors, or their employees, makes any warranty, express or implied, or assumes any legal liability or responsibility for the accuracy, completeness, or any third party's use or the results of such use of any information, apparatus, product, or process disclosed, or represents that its use would not infringe privately owned rights. Reference herein to any specific commercial product, process, or service by trade name, trademark, manufacturer, or otherwise, does not necessarily constitute or imply its endorsement, recommendation, or favoring by the United States Government or any agency thereof or its contractors or subcontractors. The views and opinions of authors expressed herein do not necessarily state or reflect those of the United States Government or any agency thereof. 


\title{
Is there a path from cuprates towards room-temperature superconductivity?
}

\author{
I. Božović ${ }^{1,2}$, J. Wu ${ }^{1}, \mathrm{X} . \mathrm{He}^{1,2}$ and A. T. Bollinger ${ }^{1}$ \\ ${ }^{1}$ Brookhaven National Laboratory \\ ${ }^{2}$ Applied Physics Department, Yale University
}

\begin{abstract}
A brief account is presented of an extensive experiment performed at Brookhaven National Laboratory, aimed at understanding the nature of high-temperature superconductivity in cuprates. Over the course of the last twelve years, over 2,000 films of the prototypical high- $T_{c}$ superconductor, $\mathrm{La}_{2-x} \mathrm{Sr}_{x} \mathrm{CuO}_{4}$, have been synthesized using atomic-layer-bylayer molecular beam epitaxy (ALL-MBE), characterized by a range of techniques, and patterned into devices. These were used to measure accurately the key physical parameters in both the superconducting and the normal states, and establish their precise dependence on doping, temperature, and external fields. The results bring in some great surprises, challenge the commonly held beliefs, rule out many theoretical models, and point to a new path for raising $T_{c}$ even further.
\end{abstract}

Keywords: high-temperature superconductivity, cuprates, molecular beam epitaxy, superfluid density, electronic nematicity

\section{Background and motivation}

Ever since Bardeen, Cooper and Schrieffer (BCS) presented their renowned theory of superconductivity $^{1}$, it has been the standard explanation of the phenomenon, in all known superconductors. This picture has been challenged by the discoveries of high-temperature superconductivity (HTS) in various cuprate compounds, by G. Bednorz and K. A. Mueller, Ching-Wu (Paul) Chu, and others, with the record critical temperature rising within just a few years by more than sixfold to reach $135 \mathrm{~K}$ (and $164 \mathrm{~K}$ under pressure). ${ }^{2-4}$ Right away, Phillip W. Anderson asserted that the superconducting state in cuprates is not just unusual, but in fact unprecedented: a BoseEinstein condensate (BEC) of charged bosons ('holons'). ${ }^{5}$ Several other prominent theorists Nevil Mott, T. D. Lee, A. F. Andreev, Pierre-Gilles De Gennes and Guy Deutscher, among others - also took this stand, as well as K. A. Mueller himself,. ${ }^{6-10}$ The plausibility of this picture has been reinforced subsequently, because experiments with cold atoms have shown that a crossover from BCS to BEC can be tuned by increasing the pairing interaction strength, and/or by making the cloud thinner and crossing over from the three-dimensional (3D) to quasi-two- 
dimensional (2D) behavior. In either case, $T_{C}$ increases and gets much higher on the BEC side. ${ }^{11-}$ 13 Thus, both the high $T_{c}$ and the 2D nature of cuprates point to BEC rather than to BCS.

Nevertheless, this view has not been universally accepted, largely because angle-resolved photoemission (ARPES) experiments have shown the presence of a Fermi Surface (FS) in metallic cuprates. ${ }^{14-16}$ This was confirmed by spectroscopic-imaging scanning-tunneling microscopy (SISTM), angle-resolved magnetoresistance oscillations (MRO), and most spectacularly, by quantum (de Haas-van Alfen and Shubnikov-de Haas) oscillations. ${ }^{17-21}$ These findings have pointed to the more-or-less standard picture in which a BCS-like superconducting state evolves upon cool-

ing from a Fermi liquid (FL) state. ${ }^{22,23}$ It seems that the majority of the researchers in the field favor this scenario - albeit with many variants insofar as the microscopic nature of the pairing interaction(s), or 'glue', is concerned — at least, on the overdoped side of the phase diagram.

This dichotomy is quite important because the physics in the two limits, BCS vs. BEC, is different both in terms of what controls $T_{c}$ and in what type of excitations, quasi-particle like or collective, deplete the condensate upon thermal agitation. Still, the debate about the nature of superconducting state in cuprates has remained an open question.

\section{The BNL high- $T_{c}$ experiment}

To address this important question, one of us (I.B.) proposed in 2004 to the US Department of Energy a project along the following lines.

In cuprates, the phase diagram on the underdoped side is quite complicated and controversial, with various types of order (charge density waves, spin density waves, etc.) observed, and many more hypothesized. Pretty much every detail, including the relevance for the HTS phenomenon of these other orders, competing or intertwined, has been hotly debated. It seemed that the only detail that almost everyone agrees upon is that at the extreme overdoped side of the phase diagram the superconducting state is of the standard BCS nature (except for the unusual d-wave symmetry of the order parameter), and that it emerges from a standard FL state. If this is the case, one could start from this well-understood fixed point and move adiabatically towards the optimum doping, measuring on the way the most relevant physical properties of the superconducting state ( $T_{c}$, the magnetic penetration depth, the coherence length) and of the normal state (the resistivity, the Hall constant, the magnetoresistance). Of course, one needs to make sure that the measured properties are intrinsic, which means that the samples must be of the highest quality, the measurements accurate, and the results robust and reproducible. Then one of two things could happen. All the physical properties may turn to evolve smoothly, in which case we would infer that the superconducting state at optimal doping is at least qualitatively similar to the BCS state. Alternatively, we may encounter some jumps and singularities, in which case all bets are off - the superconducting state at optimal doping may be of an entirely different nature, like the resonant valence bond (RVB) state of P. W. Anderson, anyons, loop currents, etc.

The project was approved and funded by U.S. Department of Energy, and the work at Brookhaven National Laboratory (BNL) started in earnest in 2005. Conceptually simple, this experiment turned out to require much technical development and innovation, and took a dozen years to complete.

\section{Technical advances}


'Tuning the doping adiabatically' translates into a need to produce many samples, with exquisite control of the chemical composition. For this, we have developed a special tool, an atomic-layerby-layer molecular beam epitaxy (ALL-MBE) system. ${ }^{24}$ It is equipped with advanced surfacescience tools that enable detailed studies of the film growth. We designed the system to be completely modular, thus achieving a very high up-time and productivity; we perform over 200 synthesis experiments every year. Thus we were able to establish the 'recipes' for synthesis of the highest-quality, single-crystal thin films of HTS cuprates and other complex oxides, with atomically smooth surfaces and interfaces. In this study, we have focused on the prototypical high- $T_{C}$ superconductor, $\mathrm{La}_{2-x} \mathrm{Sr}_{x} \mathrm{CuO}_{4}$ (LSCO), because it is the simplest and because by varying the $\mathrm{Sr}$ doping level $x$ we can cover the entire phase diagram, from insulator to superconductor to a nonsuperconducting metal. The best LSCO films show $T_{c}$ uniform to the $\pm 0.1 \mathrm{~K}$ level, across a relatively large area $\left(10 \times 10 \mathrm{~mm}^{2}\right)$. Moreover, by virtue of our 'digital' synthesis, we can engineer the samples at an atomic-layer level, optimizing them according to the needs of a particular experiment $^{25-35}$, which also turned out to be crucial for the task at hand.

In parallel, we have been developing and improving various techniques for measurements of the relevant physical properties. Important examples are the advances we made in the mutual inductance technique for measuring the magnetic penetration depth $\lambda$, a key parameter of the superconducting state. We have invented a device to suppress the thermal noise in a cryocooler to below $1 \mathrm{mK}$, an improvement by one-two orders of magnitude over the performance of commercially available units. ${ }^{36}$ Tightening the electronic, mechanical and thermal stability of the mutualinductance apparatus, we have achieved remarkable reproducibility; we have demonstrated that measurements on the same sample reproduced to within $0.1 \%$ after one year. ${ }^{37}$ We have also expanded the range of temperatures (down to $300 \mathrm{mK}$ ), frequencies (up to $50 \mathrm{MHz}$ ), and fields (up to $9 \mathrm{~T}$ ). At this point, the accuracy of our measurements of $\lambda(T)$ is not limited by the hardware, but rather by how well one knows the film thickness.

The later also turned out to be nontrivial; it took a decade of experimentation and study. By virtue of our layer-by-layer synthesis technique we know the total film thickness to the crystallographic accuracy, but the problem is in the formation of the dead layer, of unknown thickness and composition, next to the substrate, and another one next to the surface, because of the interaction with the atmosphere. To solve this problem, we protect the film by a metallic buffer layer and an overlayer. This, however, introduces another problem: the chemical potential in the active HTS material is in general different from that in the compound used for the protective layers, so there is some charge redistribution across each interface. To minimize this, we use graded doping near the two interfaces, a technique developed for growth of III-V heterostructures. Our final touch is $\mathrm{Zn} \delta$-doping in the transition layers (see Figure 1), a technique we invented and used to demonstrate that the HTS interface superconductivity in LSCO/LCO heterostructures is confined to a single $\mathrm{CuO}_{2}$ plane. ${ }^{27}$ This removes the interface superconductivity in the transition layers,

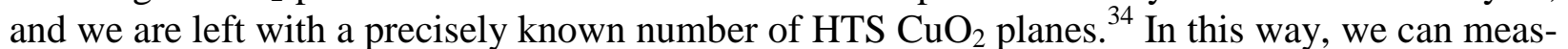
ure the absolute value of the penetration depth to better than $\pm 1 \%$ absolute accuracy. ${ }^{37}$ One could call this metrology; this is as quantitatively accurate as can be done today.

To summarize this section, we have advanced the technique of synthesis of cuprate superconductors to a new level, both in terms of film quality and by engineering the sample at the atomiclayer level. As important, by virtue of great productivity of our ALL-MBE system, we have synthesized and studied a very large sample set of over 2,000 LSCO films, perhaps without precedence in condensed matter physics. This has been decisive for the success of this project. When 
one deals with complex materials or objects, more is different; a large statistics reveals clear trends and intrinsic properties.

\section{Penetration depth study: a strange superconducting state in LSCO}

The result of "the Brookhaven HTS Experiment" that has been easiest to disclose, if not to interpret, has been a systematic dependence of $\lambda$ on doping and temperature, covering densely the entire overdoped side of the LSCO phase diagram. ${ }^{34}$ In a nutshell, the main finding is that, as we vary the doping from optimally doped all the way to non-superconducting metal, the penetration depth evolves smoothly and continuously, without any jumps. The data carry other important messages that get clearer if we plot the superfluid density per one LSCO formula unit, $N_{s}(T, p)$, which is proportional to $1 / \lambda^{2}$. In Figure 2a, one can see that, surprisingly, $N_{s}$ is essentially linear in temperature all the way to $T_{c}$, even in the most overdoped LSCO films. The same is true all the way up to the optimum doping. ${ }^{34}$ Second, in BCS theory, at $T=0$ the superfluid density $N_{s} 0$ $\equiv N_{s}(T \sqsubset 0)$ should be equal to the carrier density, so like the later $N_{s 0}$ should also increase with doping. What we see, however, is that as we increase the doping, $N_{s 0}$ decreases, all the way to zero. Third, one can express the superfluid density in the units of Kelvin to get the so-called superconducting phase stiffness. Intuitively, the later indicates the temperature scale at which thermal fluctuations of the phase of the superconducting order parameter get strong enough to destroy macroscopic superconductivity. We find that in cuprates this temperature is extremely small, roughly equal to $T_{C}$ itself. To illustrate this point, we can compare a standard BCS superconductor such as $\mathrm{Nb}$ with an LSCO sample overdoped to exactly match the $T_{c}$ of $\mathrm{Nb}$. We find that in LSCO, the phase stiffness is three orders of magnitude smaller than in $\mathrm{Nb}$ - the smallest it could possibly be. We conclude that in cuprates, the physics is dominated by massive phase fluctuations, present over a very broad temperature range, as argued by Kivelson and Emery. ${ }^{38}$ But, as seen from Figure 2b, a sharp Berezinskii-Kosterlitz-Thouless (BKT) phase transition is not observed, even in the films that are just a single unit cell thick. It can be observed only at very high ( $\mathrm{MHz}$ to $\mathrm{THz})$ frequencies. ${ }^{39-41}$

From the same $\lambda(T, p)$ data set, one can extract one more precious piece of information, the scaling of $T_{c}$ with the zero-temperature superfluid density $N_{s 0} \equiv N_{s}(T\llcorner 0)$. We have found this dependence to be smooth and essentially linear, but with a finite offset, except close to the origin where it turns to a square-root dependence. ${ }^{30}$ This is incompatible with the BCS theory, which fails spectacularly both quantitatively and qualitatively. Here, we are referring not only to the standard ('clean') BCS description, but also to its various extensions that take into account the dwave symmetry of the order parameter, as well as the effects of disorder, impurities, pair breaking, etc. While there is no space here to present the detailed arguments and evidence, let us just remark that no BCS-based approach that we have tried or seen so far is compatible with our entire data set, and that a dirty-BCS picture can also be ruled out conclusively. ${ }^{34}$

To summarize this section, we have established that the superconducting state in cuprates is very strange, and non-BCS like. In BCS theory, $T_{C}$ is essentially controlled by the phonon frequency, electron density of states near the Fermi level, and the strength of electron-phonon interaction. None of these matter here; $T_{c}$ is determined by the kinematics (the super-electron density) rather than dynamics. This, of course, resembles more what one would expect from local pairing and the BEC scenario. In fact, it has been shown by Z. Radovic and M. Vanevic (unpublished) that both the temperature and the doping dependence of $N_{s}$ can be reproduced quantitatively using a simple quasi-2D Bose gas model. 


\section{Additional evidence for preformed local pairs}

Various other independent experimental results also point to formation of local pairs in cuprates. Here, we will only mention two that we were involved with. One of this was electrolyte gating ${ }^{28}$ of ultrathin LSCO films. By varying the gate voltage $V_{G}$, we tuned the carrier density in very fine steps across the superconductor-to-insulator transition (SIT) that occurs at the critical doping $x_{c} \approx$ 0.06 , see Figure 3 . As shown in the inset, all the $R\left(T, V_{G}\right)$ curves can be scaled very well to a universal function of the new coordinate $u \alpha\left|x-x_{c}\right| T^{2 / 3}$, indicative of 2D quantum critical behavior. Notably, the SIT occurs when the film sheet resistance reaches the quantum resistance for pairs, $R_{Q}=h /(2 e)^{2}=6.5 \mathrm{k} \Omega$. (Here, $h$ is the Planck constant and $e$ the electron charge.) This is consistent with the existence of pairs on both sides of the SIT, mobile in the superconducting state and localized in the insulating state. Similar findings were also reported in thin films and bulk crystals of other cuprates. ${ }^{42-44}$

Another piece of evidence came from THz spectroscopy experiments on films of LSCO, which showed clear signs of superconducting (phase) fluctuations ${ }^{41}$ well above $T_{c}$ (at least 20-25 K) at all doping levels, see Figure 4. This corroborated similar earlier findings in $\mathrm{Bi}_{2} \mathrm{Sr}_{2} \mathrm{CaCu}_{2} \mathrm{O}_{8}$ (BSCCO) films. ${ }^{40}$ The same conclusion was reached by a number of other researchers, who studied different cuprate compounds and used different measurement techniques. ${ }^{45-51}$

\section{A paradox and a possible resolution: a two-fluid picture}

The problem with the above conclusions about local pairing and BEC-like behavior is that they collide with equally convincing evidence about the existence of the Fermi surface compounded by ARPES, STM, and quantum oscillations measurements. Of course, one possibility is that simply something is wrong, but at this point it is not obvious what. True, ARPES and STM are surface sensitive, but essentially the same Fermi surfaces are inferred from quantum oscillation experiments that probe the bulk. The (extremely) high magnetic fields used in quantum oscillation experiments could affect the electronic states and even generate new features that are absent in the ground state, but ARPES and STM measurements are performed in zero field. As for the demise of superfluid density with overdoping, it has been observed independently in LSCO by the same (mutual inductance) technique by the group of $\mathrm{T}$. Lemberger, ${ }^{52,53}$ as well as by muon spin rotation measurements on heavily overdoped Tl-2201 bulk crystals, by two different groups. ${ }^{54,55}$ In the later case, $N_{s 0}$ can be two orders of magnitude smaller than the carrier density, while at the same time spectacularly sharp quantum oscillations show without doubt that the electron mean-free path is very long (on the scale of $1,000 \AA$ or larger) and the crystals are in the ultra-clean limit. ${ }^{20,21}$ The later, by the way, leaves very little space for speculations about phase separation (electronic or otherwise), disorder, pair-breaking, etc.

One possible way out of the paradox is to assume that we are dealing with something akin to a two-fluid model, a fermion-boson mixture, where fermionic and bosonic excitations coexist, in the normal state. This separation could even occur in $k$-space, with preformed pairs originating from the states near the anti-nodes, while the nodal quasi-particles remain unpaired and fermionic. The boson-fermion ratio should vary with doping, with the bosonic fraction progressively decreasing. Assuming that superconductivity originates from the bosonic fraction, this could explain why both $T_{c}$ and $N_{s}$ decrease with doping, and at the same time, why the normal state increasingly looks like an ordinary Fermi Liquid. 
Needless to say, this is just a vague suggestion that must first be put on solid theoretical grounds, a task that is beyond the present paper. Here, we just wish to bring up one more piece of experimental evidence. It has been shown by two independent techniques that the doping dependence of the chemical potential $\mu$ in LSCO is very strange and unlike what would be expected from a simple band structure model. From undoped to optimally doped LSCO, $\mu$ stays almost constant, as demonstrated directly (and reproduced thousand-fold) by the absence of electron transfer and redistribution across the LSCO/LCO interface. On the overdoped side, however, $\mu$ drops fast and in a linear manner with doping, exactly as one would expect from a quasi-2D metal with a cylindrical Fermi surface, see Figure 5. Such a strange 'broken' $\mu(p)$ dependence is indeed indicative of formation of some more-or-less localized mid-gap states at early stages of doping, and their concomitant broadening into a wide electron band as the doping level is increased further. It is worth noting that this is exactly what has been predicted by the Dynamic Mean-Field Theory (DMFT) studies of the Mott-Hubbard model and its evolution with doping. ${ }^{56}$

Another possibly related experimental fact is that in LSCO we have observed a spontaneous breaking of the rotational symmetry in the mobile electron fluid, by angle-resolved transverse resistivity measurements. ${ }^{35}$ By other techniques, this 'electronic nematicity' has been observed in other cuprates as well as in Fe-based superconductors. New here is that we observe it in a very large portion of the $(T, p)$ phase diagram, up to the room temperature for all but the highest doping levels. This, of course, is quite unusual; since our LSCO films are tetragonal their resistivity should be isotropic in-plane. Notably also, the amplitude of nematicity - the relative degree of orthorhombic electronic anisotropy in a tetragonal crystal - decreases with doping, in line with the decrease of $T_{c}, N_{s 0}, \mu(p)$, and the Marginal Fermi Liquid features such as the T-linear component of resistivity.

\section{Summary and outlook}

To wrap this up, we have succeeded in identifying some surprising and unique features of both the normal-metal and superconducting states in LSCO. Hence, in this prototypical HTS cuprate, a strange, non-BCS-like superconducting state develops upon cooling from an equally strange metallic state that is not a simple Fermi Liquid but a Fermi Liquid Crystal. This calls for indepth theoretical studies, possibly involving new models; it is likely imperative to also take into account fluctuations properly. Experimentally, our immediate next goal is to check how universal the above findings are for various HTS cuprates as well as for Fe-based superconductors. Then, armed with these important new insights, and with our arsenal of unique synthesis techniques and characterization probes, we plan to embark on an enlightened search for new HTS materials. Obviously, we will be looking for materials in which we expect formation of local pairs at a sufficiently high density and sufficiently mobile. If such a material could be found, or engineered at the atomic level - perhaps as a metastable, or an epitaxially stabilized compound

- it is not obvious to us why one should not be able to increase $T_{c}$ beyond the current record. Time will tell.

\section{Acknowledgments}


This research was supported by the US Department of Energy, Basic Energy Sciences, Materials Sciences and Engineering Division. X.H. is supported by the Gordon and Betty Moore Foundation's EPiQS Initiative through grant GBMF4410.

\section{References}

1. Bardeen J., Cooper L. N., Schrieffer J. R.: Theory of superconductivity. Phys. Rev. 108, 1175 (1957)

2. Bednorz J. G., Muller K. A.: Possible High-T ${ }_{c}$ superconductivity in Ba-La-Cu-O system. Zeitschrift Phys. B. 64, 189 (1986)

3. Wu M. K. et al.: Superconductivity at $93 \mathrm{~K}$ in a new mixed-phase Y-Ba-Cu-O compound system at ambient pressure. Phys. Rev. Lett. 58, 908 (1987)

4. Gao, L., et al.: Superconductivity up to $164 \mathrm{~K}$ in $\mathrm{HgBa}_{2} \mathrm{Ca}_{\mathrm{m}-1} \mathrm{Cu}_{\mathrm{m}} \mathrm{O}_{2 \mathrm{~m}+2+\mathrm{d}}(\mathrm{m}=1$, 2, and 3) under quasi-hydrostatic pressures. Phys. Rev. B 50, 4260 (1994)

5. Anderson, P. W.: The resonating valence bond state in $\mathrm{La}_{2} \mathrm{CuO}_{4}$ and superconductivity. Science 235, 1196 (1987)

6. Friedberg, R., Lee, T. D.: Boson-Fermion model of superconductivity. Phys. Lett. A 138, 423 (1989)

7. Alexandrov, A. S., Mott, N. F.: Bipolarons. Rept. Prog. Phys. 57, 1197 (1994)

8. Zhao, G. M., Hunt, M. B., Keller, H., Muller, K. A.: Evidence for polaronic supercarriers in the copper oxide superconductors $\mathrm{La}_{2-\mathrm{x}} \mathrm{Sr}_{\mathrm{x}} \mathrm{CuO}_{4}$. Nature 381, 676 (1996)

9. Andreev, A. F.: Electron pairs for HTSC. JETP Letters 79, 88 (2004)

10. Deutscher, G., de Gennes, P.-G.: A spatial interpretation of emerging superconductivity in lightly doped cuprates. C. R. Physique 8, 937-41 (2007); Erratum, ibid. 9, 773 (2008)

11. Greiner, M., Regal, C. A., Jin, D. S.: Emergence of a molecular Bose-Einstein condensate from a Fermi gas. Nature 426, 537 (2003)

12. Ries, M. G., et al.: Observation of Pair Condensation in the Quasi-2D BEC-BCS Crossover. Phys. Rev. Lett. 114, 230401 (2015)

13. Sommer, A. T., Cheuk, L. W., Ku, M. J. H., Bakr, W. S., Zwierlein, M. W.: Evolution of Fermion Pairing from Three to Two Dimensions. Phys. Rev. Lett. 108, 045302 (2012)

14. Damascelli, A., Hussain, Z. and Shen, Z.-X.: Angle-resolved photoemission studies of the cuprate superconductors. Rev. Mod. Phys. 75, 473 (2003)

15. Yoshida, T. et al.: Low-energy electronic structure of the high- $T_{c}$ cuprates $\mathrm{La}_{2-x} \mathrm{Sr}_{x} \mathrm{CuO}_{4}$ studied by angle-resolved photoemission spectroscopy. J Phys.-Condens. Mat. 19,125209 (2007)

16. Ino, A., et al.: Chemical potential shift in overdoped and underdoped $\mathrm{La}_{2-x} \mathrm{Sr}_{x} \mathrm{CuO}_{4}$. Phys. Rev. Lett. 79, 2101-2104 (1997)

17. McElroy, K., et al.: Relating atomic-scale electronic phenomena to wave-like quasi-particle states in superconducting $\mathrm{Bi}_{2} \mathrm{Sr}_{2} \mathrm{CaCu}_{2} \mathrm{O}_{8+\delta}$. Nature 422, 592 (2003)

18. Hussey, N. E., et al.: Angular dependence of the c-axis normal state magnetoresistance in single crystal $\mathrm{Tl}_{2} \mathrm{Ba}_{2} \mathrm{CuO}_{6}$. Phys. Rev. Lett. 76, 122 (1996)

19. Abdel-Jawad, M., et al.: Anisotropic scattering and anomalous normal-state transport in a high-temperature superconductor. Nature Phys. 2, 821 (2006)

20. Vignolle B., et al.: Quantum oscillations in an overdoped high- $T_{c}$ superconductor. Nature 455, 952 (2008) 
21. Bangura, A. F. et al.: Fermi surface and electronic homogeneity of the overdoped cuprate superconductor $\mathrm{Tl}_{2} \mathrm{Ba}_{2} \mathrm{CuO}_{6+\delta}$ as revealed by quantum oscillations. Phys. Rev. B 82, 140501(R) (2010)

22. Laughlin, R. B.: Fermi-Liquid Computation of the Phase Diagram of High-Tc Cuprate Superconductors with an Orbital Antiferromagnetic Pseudogap. Phys. Rev. Lett. 112, 017004 (2014)

23. Laughlin, R. B.: Hartree-Fock computation of the high- $\mathrm{T}_{\mathrm{c}}$ cuprate phase diagram. Phys. Rev. B 89, 035134 (2014).

24. Bozovic, I.: Atomic-Layer Engineering of Superconducting Oxides: Yesterday, Today, Tomorrow. IEEE Trans. Appl. Superconduct. 11, 2686 (2001)

25. Bozovic, I., et al.: No mixing of superconductivity and anti-ferromagnetism in a highcritical-temperature superconductor. Nature 422, 873 (2003)

26. Gozar, A., et al.: Interface superconductivity between a metal and a Mott insulator. Nature 455, 782 (2008)

27. Logvenov, G., Gozar, A., Bozovic, I.: High-temperature superconductivity in a single copper-oxygen plane. Science 326, 699 (2009)

28. Bollinger, A. T., et al.: Superconductor-insulator transition in $\mathrm{La}_{2-\mathrm{x}} \mathrm{Sr}_{\mathrm{x}} \mathrm{CuO}_{4}$ at the pair quantum resistance. Nature 472, 458 (2011)

29. Dean, M. P. M., et al.: Spin Excitations in a single $\mathrm{La}_{2} \mathrm{CuO}_{4}$ layer. Nature Materials 11, 850 (2012)

30. Dean, M. P. M., et al.: Persistence of magnetic excitations in $\mathrm{La}_{2-\mathrm{x}} \mathrm{Sr}_{\mathrm{x}} \mathrm{CuO}_{4}$ from the undoped insulator to the heavily overdoped non-superconducting metal. Nature Materials 12, 1019 (2013)

31. Wu, J., et al.: Anomalous independence of interface superconductivity on carrier density. Nature Materials 12, 877 (2013)

32. Shi, X., et al.: Emergence of superconductivity from the dynamically heterogeneous insulating state in $\mathrm{La}_{2-\mathrm{x}} \mathrm{Sr}_{\mathrm{x}} \mathrm{CuO}_{4}$. Nature Materials 12, 47 (2013)

33. Wu J., Bollinger, A. T., Sun Y.-J., Božović, I,: Hall Effect in quantum critical chargecluster glass. Proc. Natl. Acad. Sci. USA (PNAS) 113, 4284 (2016)

34. Božović, I., He X., Wu, J., Bollinger, A. T.: Dependence of critical temperature in overdoped copper oxides on superfluid density. Nature 536, 309 (2016)

35. Wu, J., Bollinger, A. T., He X., Božović, I.: Spontaneous breaking of rotational symmetry in copper oxide superconductors. Nature (2017) in press

36. Dubuis, G., He X., Božović, I.: Ultra-thermal-stabilization of a closed cycle cryocooler. Rev. Sci. Instr. 85, 103902 (2014)

37. He X., Gozar A., Sundling R., Božović, I.: High-precision measurement of magnetic penetration depth in superconducting films. Rev. Sci. Instr. 87, 113903 (2016)

38. Emery, V. J., Kivelson, S. A.: Importance of phase fluctuations in superconductors with small superfluid density. Nature 374, 434 (1995)

39. Gasparov, V., Božović, I.: Magnetic field and temperature dependence of complex conductance of ultrathin $\mathrm{La}_{1.65} \mathrm{Sr}_{0.45} \mathrm{CuO}_{4} / \mathrm{La}_{2} \mathrm{CuO}_{4}$ films. Phys. Rev. B 86, 094523 (2012)

40. Corson, J., Mallozzi, R., Orenstein, J., Eckstein, J. N., Bozovic, I.: Vanishing of phase coherence in underdoped $\mathrm{Bi}_{2} \mathrm{Sr}_{2} \mathrm{CaCu}_{2} \mathrm{O}_{8+\delta}$. Nature 398, 221 (1999)

41. Bilbro, L. S., et al.: Temporal correlations of superconductivity above the transition temperature in $\mathrm{La}_{2-\mathrm{x}} \mathrm{Sr}_{\mathrm{x}} \mathrm{CuO}_{4}$ probed by terahertz spectroscopy. Nat. Phys. 7, 298 (2011) 
42. Leng, X., Garcia-Barriocanal, J., Bose, S., Lee, Y., Goldman, A. M.: Electrostatic control of the evolution from a superconducting phase to an insulating phase in ultrathin $\mathrm{YBa}_{2} \mathrm{Cu}_{3} \mathrm{O}_{7-\mathrm{x}}$ films. Phys. Rev. Lett. 107, 027001 (2011). Erratum Phys. Rev. Lett. 107, 039901 (2011)

43. Mandrus, D., Forro, L., Kendziora, C., Mihaly, L.: Two-dimensional electron localization in bulk single crystals of $\mathrm{Bi}_{2} \mathrm{Sr}_{2} \mathrm{Y}_{\mathrm{x}} \mathrm{Ca}_{1-\mathrm{x}} \mathrm{Cu}_{2} \mathrm{O}_{8}$. Phys. Rev. B 44, 2418(R) (1991)

44. Semba, K., Matsuda, A.: Superconductor-to-Insulator Transition and Transport Properties of Underdoped $\mathrm{YBa}_{2} \mathrm{Cu}_{3} \mathrm{O}_{\mathrm{y}}$ Crystals. Phys. Rev. Lett. 86, 496 (2001)

45. Xu, Z. A., Ong, N. P., Wang, Y., Kakeshita, T., Uchida, S.: Vortex-like excitations and the onset of superconducting phase fluctuation in underdoped $\mathrm{La}_{2-\mathrm{x}} \mathrm{Sr}_{\mathrm{x}} \mathrm{CuO}_{4}$. Nature 406, 486 (2000)

46. Wang, Y. Y., et al.: Onset of the vortex-like Nernst signal above $\mathrm{T}_{\mathrm{c}}$ in $\mathrm{La}_{2-\mathrm{x}} \mathrm{Sr}_{\mathrm{x}} \mathrm{CuO}_{4}$ and $\mathrm{Bi}_{2} \mathrm{Sr}_{2-y} \mathrm{LaCuO}_{6}$. Phys. Rev. B 64, 224519 (2001)

47. Wang, Y., Li, L., Ong, N. P.: Nernst effect in high-T ${ }_{c}$ superconductors. Phys. Rev. B 73, 024510 (2006)

48. Li, L. et al.: Diamagnetism and Cooper pairing above $\mathrm{T}_{\mathrm{C}}$ in cuprates. Phys. Rev. B 81, 054510 (2010)

49. Grbić, M. S., et al.: Temperature range of superconducting fluctuations above $\mathrm{T}_{\mathrm{c}}$ in $\mathrm{YBa}_{2} \mathrm{Cu}_{3} \mathrm{O}_{7-\delta}$ single crystals. Phys. Rev. B 83, 144508 (2011)

50. Uykur, E., Tanaka, K., Masui, T., Miyasaka, S., Tajima, S.: Persistence of the superconducting condensate far above the critical temperature of $\mathrm{YBa}_{2}(\mathrm{Cu}, \mathrm{Zn})_{3} \mathrm{O}_{\mathrm{y}}$ revealed by caxis optical conductivity measurements for several Zn concentrations and carrier doping levels. Phys. Rev. Lett. 112, 127003 (2014)

51. Kondo, T. et al.: Point nodes persisting far beyond $\mathrm{T}_{\mathrm{c}}$ in Bi2212. Nature Commun. 6, 7699 (2015)

52. Lemberger, T. R., Hetel, I., Tsukada, A., Naito, M.: Anomalously sharp superconducting transitions in overdoped $\mathrm{La}_{2-x} \mathrm{Sr}_{x} \mathrm{CuO}_{4}$ films. Phys. Rev. B 82, 214513 (2010)

53. Lemberger, T. R., Hetel, I., Tsukada, A., Naito, M., Randeria, M.: Superconductor-to-metal quantum phase transition in overdoped $\mathrm{La}_{2-x} \mathrm{Sr}_{x} \mathrm{CuO}_{4}$. Phys. Rev. B 83, 140507 (2011)

54. Uemura, Y. J., et al.: Magnetic-field penetration depth in $\mathrm{Tl}_{2} \mathrm{Ba}_{2} \mathrm{CuO}_{6+\delta}$ in the overdoped regime. Nature 364, 605 (1993)

55. Niedermayer, C., et al.: Muon spin rotation study of the correlation between $T_{c}$ and $n_{s} / m *$ in overdoped $\mathrm{Tl}_{2} \mathrm{Ba}_{2} \mathrm{CuO}_{6+\delta}$. Phys. Rev. Lett. 71, 1764 (1993)

56. Kotliar, G., Vollhardt, D., Strongly correlated materials: Insights from dynamical meanfield theory, Phys. Today 57, 53 (2004) 


\begin{tabular}{|c|}
\hline $10 \times \mathrm{La}_{1.60} \mathrm{Sr}_{0.40} \mathrm{CuO}_{4}$ \\
\hline $\mathrm{La}_{1.65} \mathrm{Sr}_{0.35} \mathrm{Cu}_{0.97} \mathrm{Zn}_{0.03} \mathrm{O}_{4}$ \\
\hline $\mathrm{La}_{1.70} \mathrm{Sr}_{0.30} \mathrm{Cu}_{0.97} \mathrm{Zn}_{0.03} \mathrm{O}_{4}$ \\
\hline $10 \times \mathrm{La}_{1.75} \mathrm{Sr}_{0.25} \mathrm{CuO}_{4}$ \\
\hline $\mathrm{La}_{1.70} \mathrm{Sr}_{0.30} \mathrm{Cu}_{0.97} \mathrm{Zn}_{0.03} \mathrm{O}_{4}$ \\
\hline $\mathrm{La}_{1.65} \mathrm{Sr}_{0.35} \mathrm{Cu}_{0.97} \mathrm{Zn}_{0.03} \mathrm{O}_{4}$ \\
\hline $10 \times \mathrm{La}_{1.60} \mathrm{Sr}_{0.40} \mathrm{CuO}_{4}$ \\
\hline LSAO SUBSTRATE \\
\hline
\end{tabular}

Figure 1. Schematics of atomic-layer sample engineering to isolate a HTS layer of a known thickness. ${ }^{34}$ 

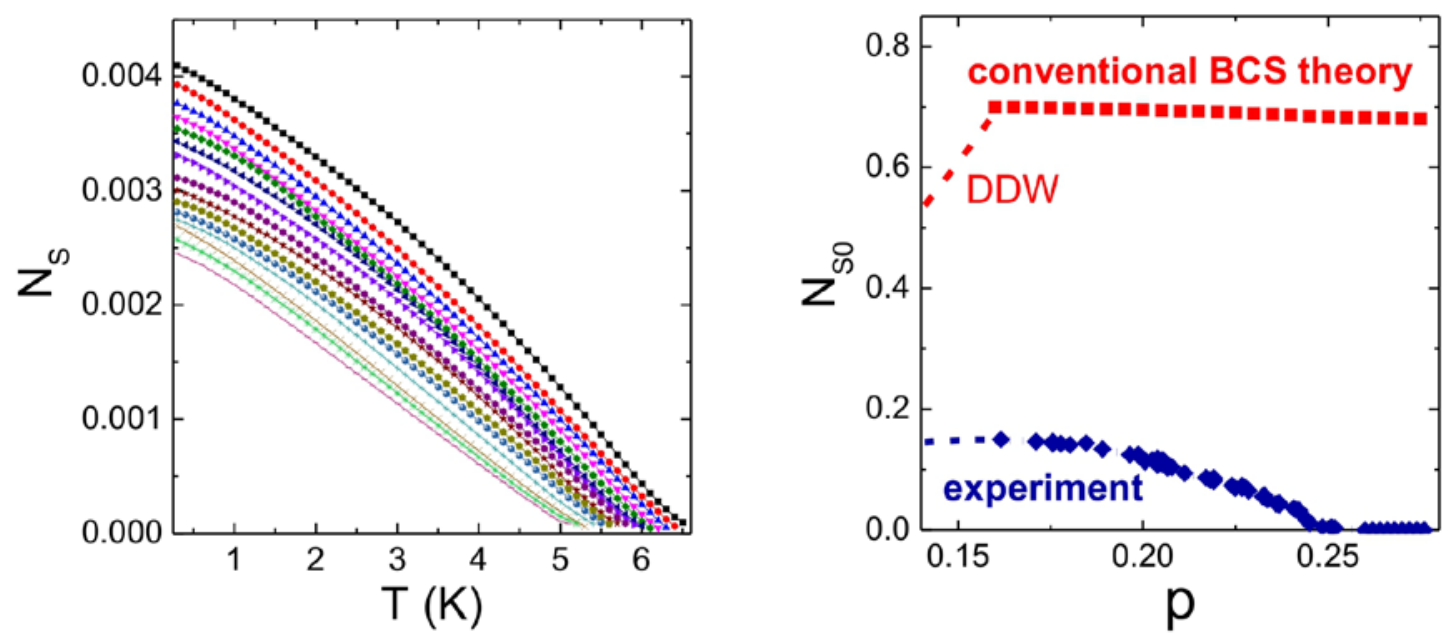

Figure 2. Left panel: Temperature and doping dependence of the superfluid density $N_{s}$ as a function of temperature and doping, in heavily overdoped LSCO films. ${ }^{34}$ Right panel: the doping dependence of $N_{s 0} \equiv N_{s}(T \sqsubset 0)$. Blue diamonds: our experimental data. Red line: the dependence expected from the BCS theory. ${ }^{23}$ 


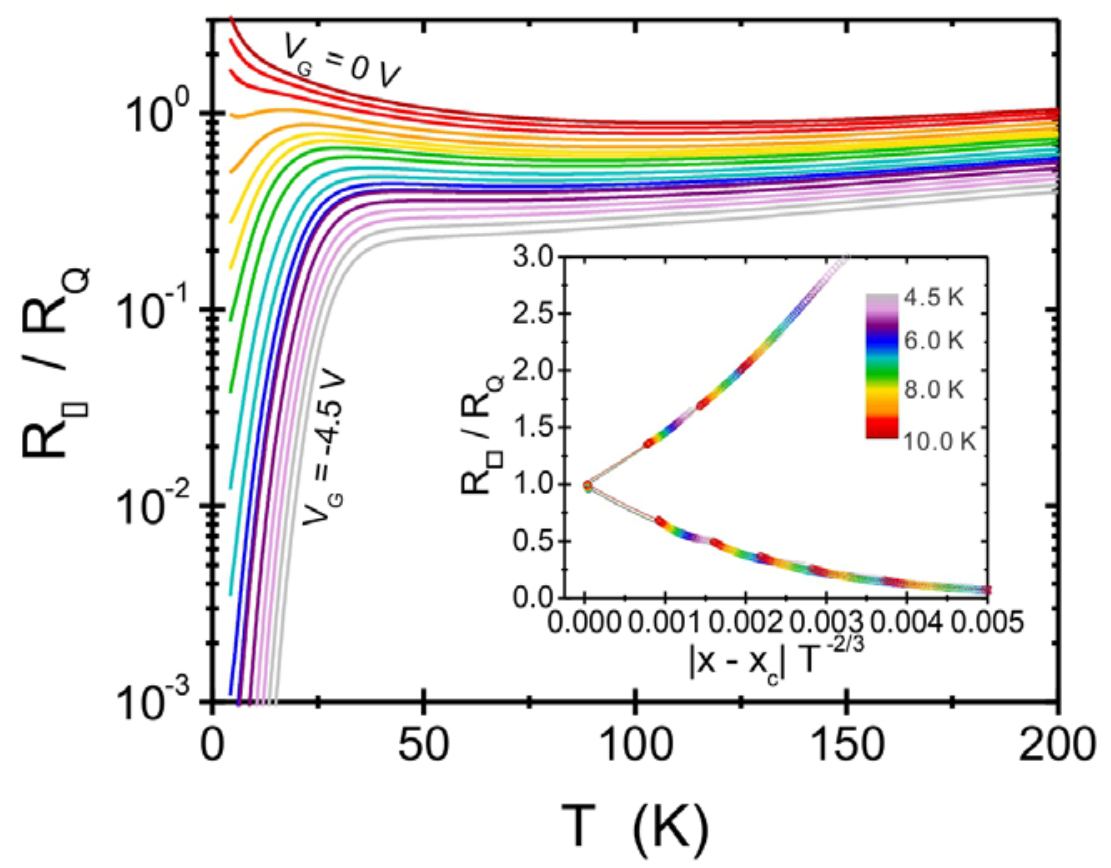

Figure 3. Electrolyte gating of a one unit cell thick LSCO layer. ${ }^{28}$ Inset: The same data rescaled against $u=\left|x-x_{C}\right| / T^{2 / 3}$. 


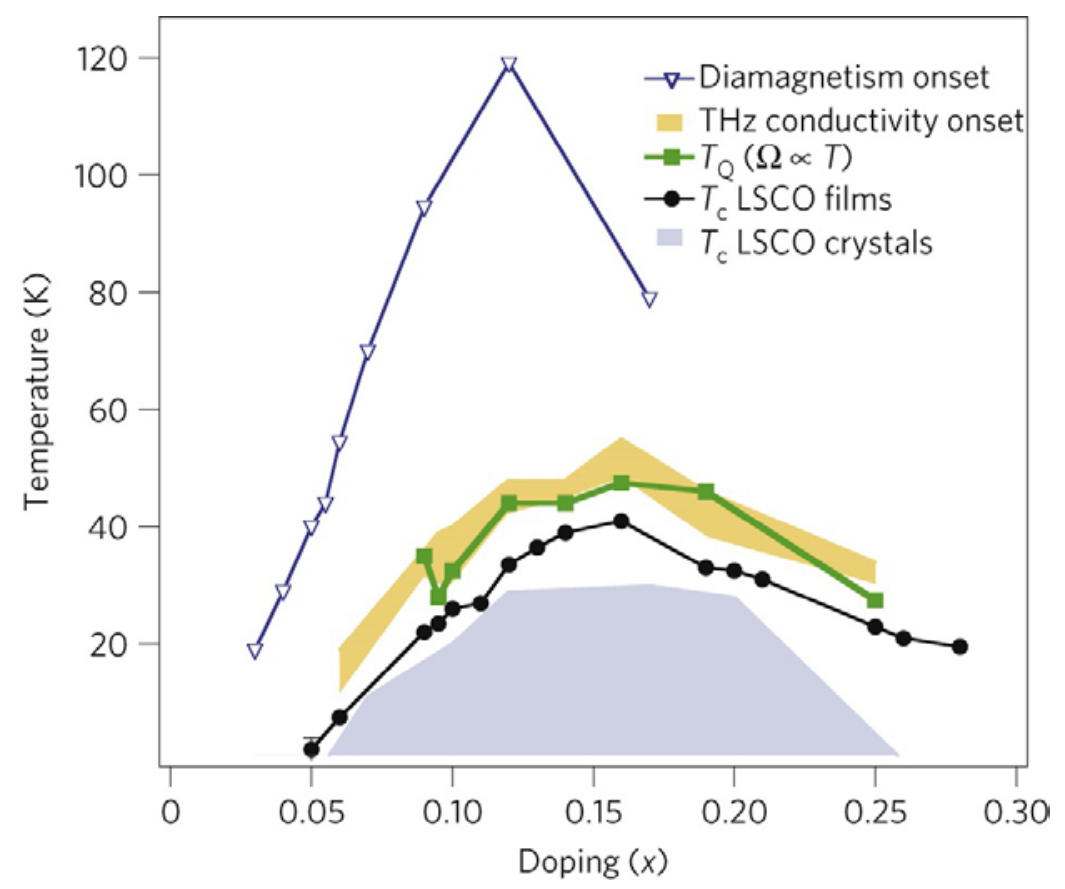

Figure 4. Superconducting fluctuations in LSCO seen well above $T_{c}$ in THz spectroscopy. ${ }^{41}$ 


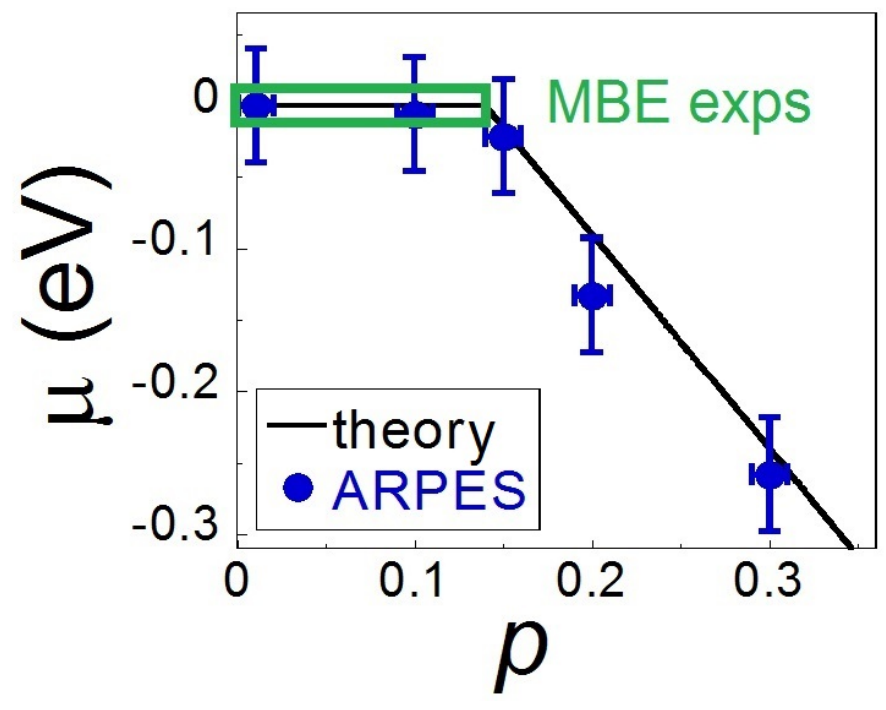

Figure 5. A strange ‘broken’ doping dependence of the chemical potential in LSCO. ${ }^{25,31}$ 